\title{
Yacon (Smallanthus sonchifolius) Leaf Extract Attenuates Hyperglycemia and Skeletal Muscle Oxidative Stress and Inflammation in Diabetic Rats
}

\author{
Klinsmann Carolo dos Santos, ${ }^{1,2}$ Bianca Guerra Bueno, ${ }^{1}$ Luana Ferreira Pereira, ${ }^{1}$ \\ Fabiane Valentini Francisqueti, ${ }^{1}$ Mariana Gobbo Braz, ${ }^{1}$ Lahis Fernandes Bincoleto, ${ }^{1}$ \\ Lilian Xavier da Silva, ${ }^{3}$ Ana Lúcia A. Ferreira, ${ }^{1}$ Ana Cláudia de Melo Stevanato Nakamune, ${ }^{3}$ \\ C.-Y. Oliver Chen, ${ }^{2}$ Jeffrey B. Blumberg, ${ }^{2}$ and Camila Renata Corrêa ${ }^{1}$ \\ ${ }^{1}$ São Paulo State University (UNESP), Medical School, Botucatu, SP, Brazil \\ ${ }^{2}$ Antioxidants Research Laboratory, Jean Mayer USDA Human Nutrition Research Center on Aging, Tufts University, \\ Boston, MA, USA \\ ${ }^{3}$ São Paulo State University (UNESP), Dentistry School, Araçatuba, SP, Brazil
}

Correspondence should be addressed to Camila Renata Corrêa; correa.camila9@gmail.com

Received 15 March 2017; Revised 6 June 2017; Accepted 12 June 2017; Published 20 July 2017

Academic Editor: Luigi Milella

Copyright (C) 2017 Klinsmann Carolo dos Santos et al. This is an open access article distributed under the Creative Commons Attribution License, which permits unrestricted use, distribution, and reproduction in any medium, provided the original work is properly cited.

\begin{abstract}
The effects of hydroethanolic extract of Yacon leaves (HEYL) on antioxidant, glycemic, and inflammatory biomarkers were tested in diabetic rats. Outcome parameters included glucose, insulin, interleukin-6 (IL-6), and hydrophilic antioxidant capacity (HAC) in serum and IL-6, HAC, malondialdehyde (MDA), superoxide dismutase (SOD), catalase (CAT), and glutathione peroxidase (GPx) in soleus. The rats (10/group) were divided as follows: C, controls; C + Y, HEYL treated; DM, diabetic controls; and DM + Y, diabetic rats treated with HEYL. Diabetes mellitus was induced by administration of streptozotocin. $\mathrm{C}+\mathrm{Y}$ and $\mathrm{DM}+\mathrm{Y}$ groups received $100 \mathrm{mg} / \mathrm{kg}$ HEYL daily via gavage for $30 \mathrm{~d}$. Hyperglycemia was improved in the DM + Y versus DM group. Insulin was reduced in DM versus C group. DM rats had higher IL- 6 and MDA and lower HAC in the soleus muscle. HEYL treatment decreased IL- 6 and MDA and increased HAC in DM rats. DM + Y rats had the highest CAT activity versus the other groups; GPx was higher in C + $\mathrm{Y}$ and DM + Y versus their respective controls. The apparent benefit of HEYL may be mediated via improving glucoregulation and ameliorating oxidative stress and inflammation, particularly in diabetic rats.
\end{abstract}

\section{Introduction}

Type 1 diabetes mellitus (T1DM) occurs by autoimmunemediated destruction of pancreatic $\beta$-cells, leading to insulin deficiency and loss of glycemic control. The hyperglycemia that occurs in diabetes increases the production of reactive oxygen species (ROS) and weakens antioxidant defense, resulting in enhanced oxidative stress [1]. ROS mediate several biochemical and molecular pathways that can exacerbate oxidative stress [2], such as activating the transcription factor nuclear factor kappa B (NF- $\kappa \mathrm{B})$, which increases the transcription of inflammatory cytokines and chemokines [3] promoting inflammation. Moreover, uncontrolled ROS generation could also attack the cellular proteins, lipids, and nucleic acids leading to cellular dysfunction including loss of energy metabolism, alteration on cell signaling and cell cycle control, mutations, and inflammation. In addition, it plays a role in several pathological processes in skeletal muscle [4]. These reactive species are important signaling molecules necessary for muscle function and for adaptive response to stress [5]. However, overproduction of ROS and decrease of the antioxidant defense have negative impact on muscle function, as impaired muscle growth and strength and altered metabolic capacity [6]. 
Medicinal plants are widely used as alternative therapeutics for the prevention or treatment of diseases. Recently, great attention has been paid to the use of natural compounds, due to their nutritional and pharmacological characteristics [7].

Yacon (Smallanthus sonchifolius [Poepp. \& Endl.] H. Robinson, Asteraceae) is a native Andean plant cultivated for its tubers, which are commonly used as a food in South America. Some studies have reported the presence of large amounts of phenolic compounds in extracts from Yacon leaves and tubers, mainly chlorogenic, protocatechuic, ferulic, rosmarinic, gallic, gentisic, and caffeic acids and their derivatives [8]. Evidence has also emerged about the antioxidant activity [9], protective effects on oxidative damage and glucose metabolism in rat hepatocytes, and insulin-like effects of Yacon leaf extracts $[10,11]$.

Antioxidant compounds have long been known to diminish inflammatory and oxidative stress responses. In addition, antioxidants scavenge ROS and increase the capacity of the antioxidant defense enzyme system [2]. Therefore, antioxidants can help diminish oxidative damage and inflammation and slow or prevent the progression of diabetic complications.

Natural products are the groundwork of preventing and curing several diseases. Moreover, ethnopharmacological knowledge is one attractive way to enhance the probability of success in new drug-finding efforts. Regarding diabetic complications, plants that can be effectively used based on their therapeutic applications, for example, for diabetes, are worthy of special attention, and studies are needed regarding their side effects, the ability to maintain normal levels of glycemia, and their possible control on oxidative stress and inflammation. Considering the complications of type 1 diabetes and previous data on Yacon's activities, the present study was undertaken to elucidate the antioxidant, anti-inflammatory, and antihyperglycemic activity of hydroethanolic extract from S. sonchifolius leaves (HEYL) in the serum and skeletal muscle of STZ-induced diabetic rats.

\section{Materials and Methods}

2.1. Plant Material and Extract Preparation. The leaves of S. sonchifolius were collected in June (2014) by Klinsmann Carolo dos Santos in Curitiba, PR, Brazil. The specimen was provided by Dr. Átila Francisco Mógor from the Department of Plant Science and Crop Protection, Federal University of Paraná, Curitiba, Paraná, Brazil, and identified by Dr. Lin Chau Ming from São Paulo State University (UNESP), School of Agriculture, Botucatu, SP, Brazil, and the voucher specimen was deposited to the Herbarium at the São Paulo State University (UNESP), Institute of Biosciences, Botucatu, SP, Brazil, under the register 32752, for future reference. The leaves from $S$. sonchifolius were dried for seven days at $50^{\circ} \mathrm{C}$, powdered $(3 \mu \mathrm{m})$, and subjected to percolation at room temperature using a mixture of ethanol: $\mathrm{H}_{2} \mathrm{O}$ $(7: 3, \mathrm{v} / \mathrm{v})$ with a flux of $2.0 \mathrm{~mL} / \mathrm{min} / \mathrm{kg}$. The solvents were evaporated to dryness under a low pressure $\left(45^{\circ} \mathrm{C}\right)$ using rotary evaporator in vacuum system to afford the crude HEYL.
2.2. Characterization of Phenolics. For the characterization of phenolics, $10 \mathrm{mg}$ HEYL was reconstituted in $1 \mathrm{~mL}$ methanol, followed by acidic hydrolysis with $1 \mathrm{~mL}$ of $2.4 \mathrm{M} \mathrm{HCl}$ at $80^{\circ} \mathrm{C}$ for $2 \mathrm{~h}$ in the dark. After the incubation, the solution was filtered through a $0.45 \mu \mathrm{m}$ nylon membrane (Millipore Corp., Bedford, MA) and then injected onto a HPLC system equipped with a Zorbax SB-C18 column $(4.6 \times$ $250 \mathrm{~mm}, 3.5 \mu \mathrm{m})$ and a CoulArray 5600A electrochemical detector (ESA Inc., Chelmsford, MA). Phenolic acids and flavonoids were quantified according to the method of Li et al. (2009) [12]. The limits of quantitation for phenolic acid and flavonoids were $1 \mathrm{ng}$ on column. The linearity of calibration curves of authentic standards with concentrations ranging from 0.01 to $2 \mathrm{ng} / \mathrm{ml}$ was at least $\geq 0.991$. The identification of each compound was based on a comparison of the retention time and electrochemical response of the authenticated standards. The results are expressed in $\mu \mathrm{g} / 100 \mathrm{mg}$ HEYL.

2.3. Dose-Response Profile of HEYL Treatment. To establish a dose-response profile for the antihyperglycemic activity of Yacon leaves, we used varying doses of HEYL $(25,50$, and $100 \mathrm{mg} / \mathrm{kg}$ body weight/day constituted in $1 \mathrm{~mL}$ of $0.9 \%$ saline) to identify the lowest dose that could elicit an optimal antihyperglycemic effect. Fifteen male Wistar rats, $60 \mathrm{~d}$ of age, were maintained in an environmentally controlled room $(22$ $\pm 3^{\circ} \mathrm{C}$; 12 -hour light/dark cycle and relative humidity of $60 \pm$ $5 \%$ ) and were fed with a standard rat pellet diet (Purina Ltd., Campinas, SP, Brazil) and water ad libitum. The animals were randomly assigned to one of three groups: HEYL 25; HEYL 50; and HEYL 100. Diabetes mellitus was induced by one i.p. administration of streptozotocin (STZ; $40 \mathrm{mg} /$ body weight), and the animals received HEYL for gavage for 2 weeks after the establishment of diabetic condition. We found that after 2 weeks of the treatment, the highest dose showed the most potent hyperglycemic effect in STZ model of diabetes. Thus, the subsequent experiments with HEYL were carried out with the dose of $100 \mathrm{mg} / \mathrm{kg}$ administered orally.

2.4. Animals and Experimental Groups. Forty male Wistar rats, $60 \mathrm{~d}$ of age, were maintained in an environmentally controlled room $\left(22 \pm 3^{\circ} \mathrm{C}\right.$; 12 -hour light/dark cycle and relative humidity of $60 \pm 5 \%$ ) and were fed with a standard rat pellet diet (Purina Ltd., Campinas, SP, Brazil) and water ad libitum. The experimental protocol was approved by the Ethics Committee on the Use of Animals (CEUA) at the Botucatu Medical School, São Paulo State University (UNESP) under number 1082-2014 (approved in April 24, 2014). The animals were randomly assigned to one of four groups $(n=10)$ : $\mathrm{C}$ (control group): normal rats; $\mathrm{C}+\mathrm{Y}$ : normal rats receiving HEYL; DM: diabetic rats; and DM+Y: diabetics rats receiving HEYL. Diabetes mellitus was induced by i.p. administration of streptozotocin for one time (STZ; $40 \mathrm{mg} /$ body weight). Blood glucose was measured $48 \mathrm{~h}$ and 7 days after the STZ administration. The animals with blood glucose greater than $250 \mathrm{mg} / \mathrm{dL}$ were considered diabetic. The animals received HEYL $(100 \mathrm{mg} / \mathrm{kg}$ body weight/day constituted in $1 \mathrm{~mL}$ of $0.9 \%$ saline) for gavage for 30 days after the 7 th day of established diabetic condition. Control animals were given the same volume of saline. The animals were fasted overnight and killed by decapitation 
after anaesthesia with ketamine $(50 \mathrm{mg} / \mathrm{kg})$ and xylazine $(0.5 \mathrm{mg} / \mathrm{kg})$ by intraperitoneal injection, and all efforts were made to minimize suffering. Blood was collected in tubes and then centrifuged at $3500 \mathrm{rpm} \times \mathrm{g}$. The serum and soleus muscle were collected and stored at $-80^{\circ} \mathrm{C}$ until analysis.

2.5. Preparation of the Soleus Muscle for Analysis. Soleus muscle was weighed $(100 \mathrm{mg})$ and homogenized in $1.0 \mathrm{~mL}$ cold PBS (pH 7.4) using ULTRA-TURRAX ${ }^{\circledR}$ T25 basic IKA ${ }^{\circledR}$ Werke Staufen/Germany. After centrifugation at $800 \times \mathrm{g}$ at $4^{\circ} \mathrm{C}$ for $10 \mathrm{~min}$, the supernatant was collected for malondialdehyde (MDA) and IL-6 determinations. For the antioxidant enzymes determination, $100 \mathrm{mg}$ soleus muscle was homogenized $(1: 10 \mathrm{v} / \mathrm{v})$ in $\mathrm{KH}_{2} \mathrm{PO}_{4}(10 \mathrm{mmol} / \mathrm{L}) / \mathrm{KCl}$ $(120 \mathrm{mmol} / \mathrm{L}), \mathrm{pH} 7.4$, and centrifuged at $2.000 \times \mathrm{g}$ for $20 \mathrm{~min}$.

2.6. Biochemical Measurements in Serum and Soleus Muscle. An enzymatic colorimetric kit was used to measure serum glucose (Bioclin ${ }^{\circledR}$, Belo Horizonte, Minas Gerais, Brazil). Insulin (Immuno-Biological Laboratories, Inc.) and IL-6 ( $R \& D$ Systems, Inc.) were measured by an immunoassay, using a microplate reader (Spectra Max 190; Molecular Devices).

2.7. Pancreatic Beta-Cell Function. Pancreatic beta-cell function was determined using the index of homeostasis model assessment (HOMA) [13] using the following formula: HOMA-BETA (Homeostasis Model Assessment BetaCell Function $)=20 \times$ Fasting Insulin $(\mu \mathrm{U} / \mathrm{mL}) /$ Fasting Glucose $(\mathrm{mM})-3,5$.

2.8. Malondialdehyde (MDA) Analysis in Soleus Muscle. A $100 \mu \mathrm{L}$ aliquot of soleus muscle homogenate was used for MDA analysis. Briefly, we added $700 \mu \mathrm{L}$ of $1 \%$ orthophosphoric acid and $200 \mu \mathrm{L}$ of thiobarbituric acid $(42 \mathrm{mM})$ to the sample and then boiled it for $60 \mathrm{~min}$ in a water bath; the sample was cooled on ice immediately after that. Two hundred $\mu \mathrm{L}$ was transferred to a $2 \mathrm{~mL}$ tube containing $200 \mu \mathrm{L}$ sodium hydroxide-methanol $(1: 12 \mathrm{v} / \mathrm{v})$. The sample was vortex-mixed for $10 \mathrm{~s}$ and centrifuged for $3 \mathrm{~min}$ at $13,000 \times \mathrm{g}$. The supernatant $(200 \mu \mathrm{L})$ was transferred to a $300 \mu \mathrm{L}$ glass vial and $50 \mu \mathrm{L}$ injected onto the column. The HPLC was a Shimadzu LC-10AD system (Kyoto, Japan) equipped with a C18 Luna column $(5 \mu \mathrm{m}, 150 \times 4.60 \mathrm{~mm}$, Phenomenex Inc., Torrance, CA, USA), a Shimadzu RF-535 fluorescence detector (excitation: $525 \mathrm{~nm}$, emission $551 \mathrm{~nm}$ ), and $0.5 \mathrm{~mL} / \mathrm{min}$ flow of phosphate buffer $\left(\mathrm{KH}_{2} \mathrm{PO}_{4} 1 \mathrm{mM}, \mathrm{pH}\right.$ 6.8) [14]. MDA was quantified by area determination of the peaks in the chromatograms relative to a standard curve of known concentrations.

2.9. Measurement the Hydrophilic Antioxidant Capacity (HAC) in Serum and Soleus Muscle. The hydrophilic antioxidant capacity was determined fluorometrically, as described by Beretta et al. (2006) [15] using a VICTOR X2 reader (Perkin Elmer, Boston, MA). The antioxidant activity was quantitated by comparing the area under the curve relating to the oxidation kinetics of the suspension phosphatidylcholine
(PC), which was used as reference biological matrix. The peroxyl radical 2',2'-azobis-(2-amidinopropane) dihydrochloride $(\mathrm{AAPH})$ was used as an initiator of the reaction. The results represent the percent inhibition (4,4 difluoro-5-(4phenyl 1-3 butadiene)-4-bora-3,4-diaza-s-indacene) (BODIPY) 581/591 plasma with respect to the control sample of BODIPY 581/591 PC liposome. All analyses were performed in triplicate. The results are reported as percentage of protection.

2.10. Antioxidant Enzymes Activity Evaluation in Soleus Muscle. Superoxide dismutase activity was measured based on the inhibition of a superoxide radical reaction with pyrogallol and the absorbance values were measured at $420 \mathrm{~nm}$ [16]. The values are expressed as units per milligram of protein. Catalase activity was evaluated by following the decrease in the levels of hydrogen peroxide. The absorbance values were measured at $240 \mathrm{~nm}$ [17]. The activity is expressed as pmole of $\mathrm{H}_{2} \mathrm{O}_{2}$ reduced/min/mg protein. Glutathione peroxidase activity was measured by following $\beta$-nicotinamide adenine dinucleotide phosphate (NADPH) oxidation at $340 \mathrm{~nm}$ as described by Flohé and Günzler (1984) [18]; the results were expressed as $\mu \mathrm{mol}$ hydroperoxide reduced/min/mg protein. Protein was quantified based on Lowry's method [19], using bovine serum albumin as the standard.

2.11. Statistical Analysis. Results are expressed as mean and standard error of the mean (SEM), and significance was calculated by two-way ANOVA followed by Holm-Sidak method. The software used was SigmaStat version 3.5 for Windows (Systat Software, Inc., San Jose, CA, USA). Differences were considered significant at $P<0.05$.

\section{Results}

3.1. Glycemia, Insulin, and HOMA-BETA. STZ-induced diabetic rats (DM and $\mathrm{DM}+\mathrm{Y}$ ) showed 3.25- and 3.08-fold, respectively, higher blood glucose levels than the control groups (Figure 1(a)) in the beginning of the experiment $(7 \mathrm{~d}$ after administration of STZ). After treatment with HEYL, the $\mathrm{DM}+\mathrm{Y}$ animals showed reduction of glycemia to values similar to the controls (Figure 1(b)). The insulin was lower in DM when compared with the control groups (Figure 1(c)). The same was found for HOMA-BETA; DM presented the lowest values when compared to $\mathrm{C}$ and $\mathrm{DM}+\mathrm{Y}$, whereas the treated DM group presented increase of HOMA-BETA (Figure 1(d)).

3.2. Characterization of Phenolics. Ten phenolics in the hydroethanolic extract of Yacon were quantified using a HPLC-ECD method. The active principles with their concentrations, retention time $(\mathrm{RT})$, and peak area $(\mu \mathrm{C})$ are presented in Table 1.

3.3. Antioxidant Enzymes and Lipoperoxidation Marker in Soleus Muscle. The treatment with Yacon leaves in DM + Y decreased MDA (Figure 3(d)) in soleus muscle when compared to DM, which presented the highest values for this variable. $\mathrm{DM}+\mathrm{Y}$ group presented the highest catalase 


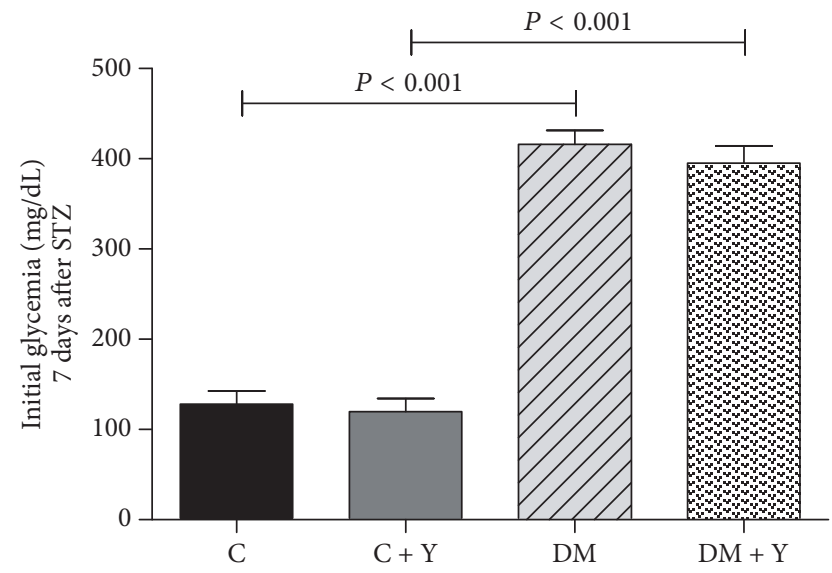

(a)

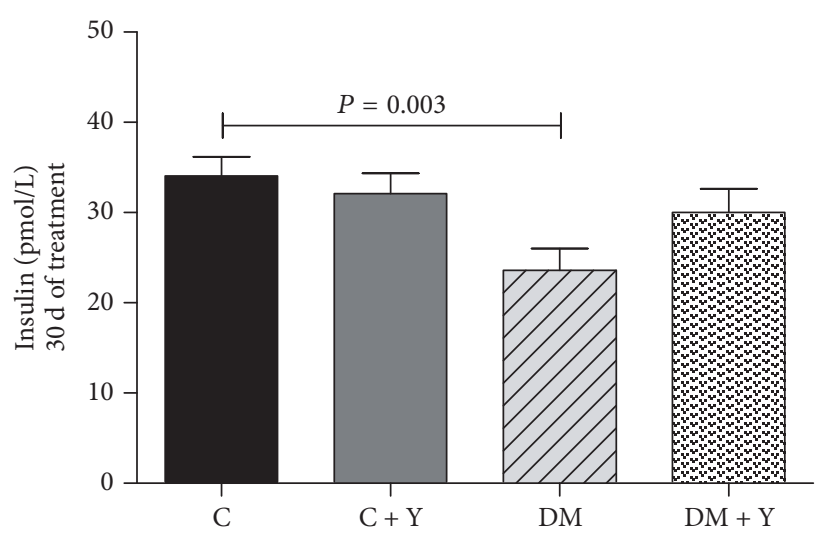

(c)

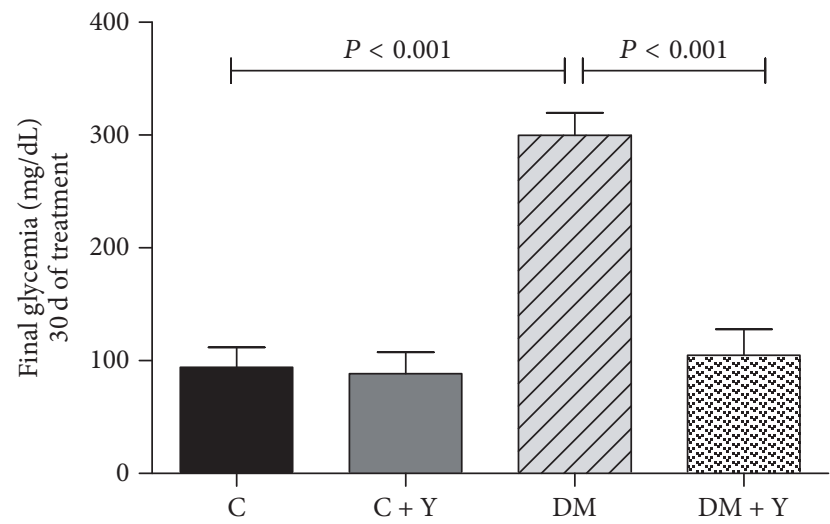

(b)

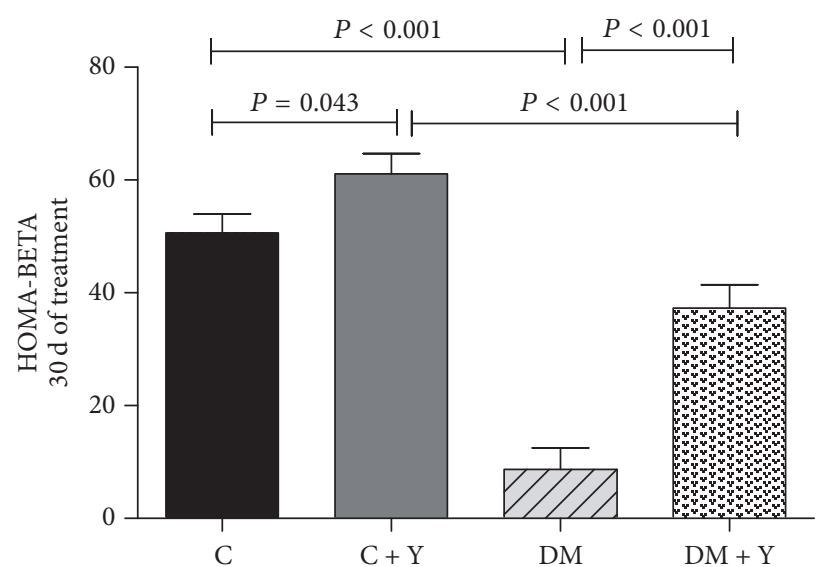

(d)

FIgURE 1: (a) Initial glycemia (7 d after STZ administration); (b) final glycemia (30 d of treatment); (c) insulin (30 d of treatment); (d) HOMABETA ( $30 \mathrm{~d}$ of treatment) of the different experimental groups. C (control group): normal rats; C + Y: normal rats receiving HEYL; DM: diabetic rats; and DM + Y: diabetics rats receiving HEYL. The results are expressed as the mean \pm SEM.

TABLE 1: Phenolic content of hydroethanolic extract of Yacon leaves.

\begin{tabular}{lccc}
\hline RT $(\min )$ & Name & Peak area $(\mu \mathrm{C})$ & Concentration \\
\hline 15.29 & Protocatechuic acid & 6.98 & 10.11 \\
25.83 & Gentisic acid & 8.78 & 7.64 \\
33.13 & Chlorogenic acid & 2.30 & 8.17 \\
35.97 & Vanillic acid & 3.08 & 1.34 \\
37.65 & Caffeic acid & 66.10 & 27.56 \\
42.25 & Epicatechin & 2.20 & 5.11 \\
46.67 & p-Coumaric acid & 19.50 & 169.81 \\
49.78 & Ferulic acid & 7.05 & 13.21 \\
50.43 & Sinapic acid & 6.41 & 4.68 \\
75.35 & Quercetin & 128.0 & 399.00 \\
\hline
\end{tabular}

RT: retention time. Concentration is expressed in $\mu \mathrm{g} / 100 \mathrm{mg}$ of HEYL. 


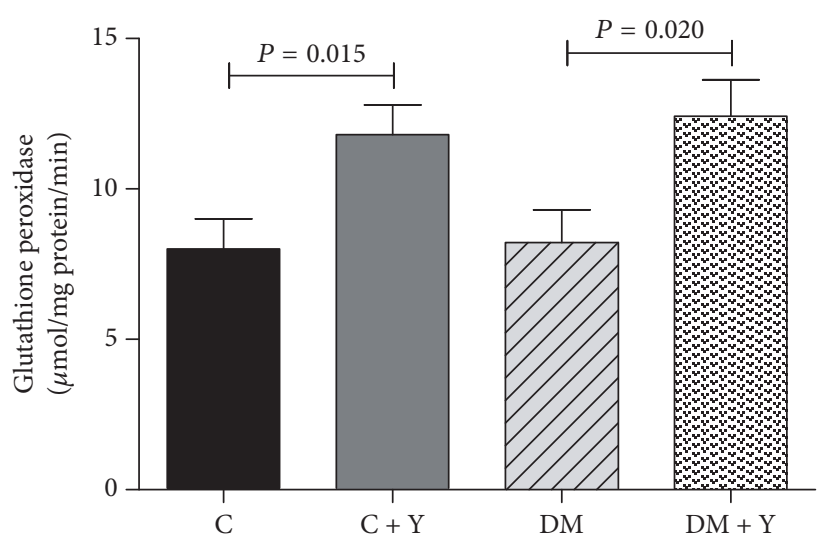

(a)

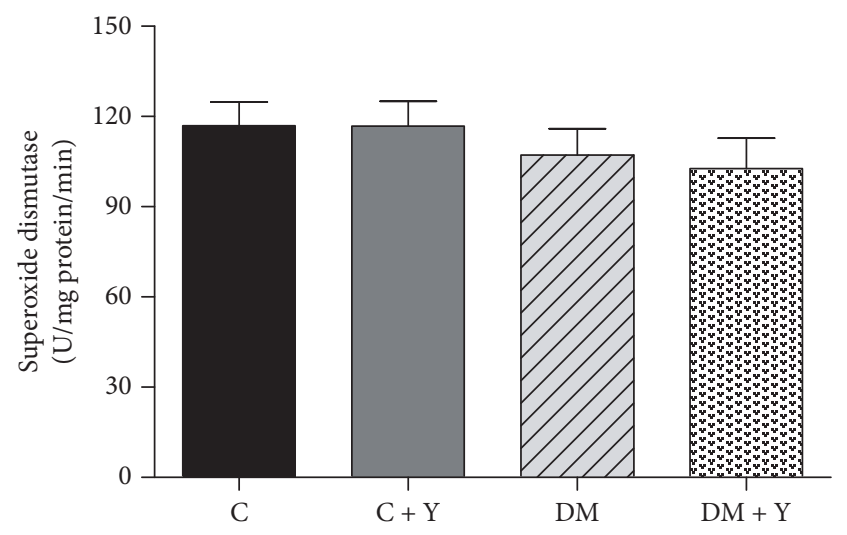

(c)

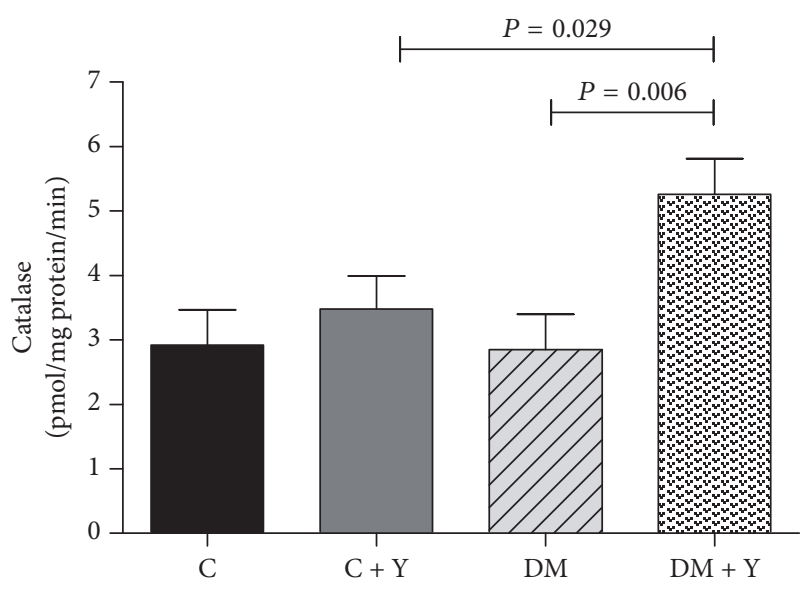

(b)

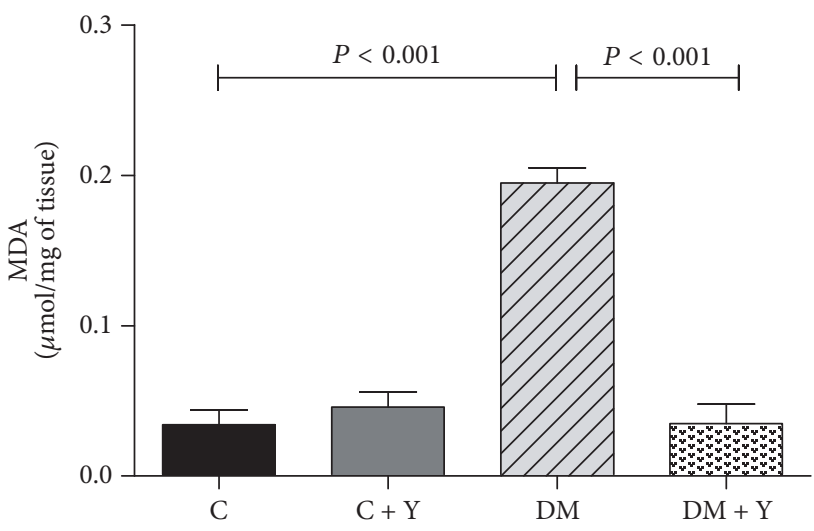

(d)

FIGURE 2: (a) Catalase (CAT) activity; (b) superoxide dismutase (SOD) activity; (c) glutathione peroxidase (GPx) activity; (d) MDA concentration in soleus of the different experimental groups. C (control group): normal rats; C + Y: normal rats receiving HEYL; DM: diabetic rats; and DM + Y: diabetics rats receiving HEYL. The results are expressed as the mean \pm SEM.

activity among the groups (Figure 3(b)), whereas the GPX (Figure 3(a)) was higher in $\mathrm{C}+\mathrm{Y}$ and $\mathrm{DM}+\mathrm{Y}$. No significant difference was found for SOD (Figure 3(c)) among the groups.

\subsection{Hydrophilic Antioxidant Capacity (HAC) and IL-6 in} Serum and Soleus Muscle. There were no significant differences for plasma IL-6 (Figure 2(c)). Plasma HAC was higher in $\mathrm{C}+\mathrm{Y}$ when compared to $\mathrm{C}$ group, although, when evaluated in soleus muscle, a 2.2-fold increase of IL-6, with a $29.1 \%$ decrease of HAC, was observed in DM group compared to $\mathrm{C}$ group (Figure 2). HEYL promoted decrease of IL-6 and increase of HAC in DM $+\mathrm{Y}$ group when compared to untreated group.

\section{Discussion}

Streptozotocin (STZ) is a widely used chemical for the induction of experimental diabetes [20]. Type 1 diabetes can be induced in rodents by a single STZ injection [21]. All these STZ-induced diabetic animal models have been useful in elucidating the mechanisms of diabetic pathogenesis and in screening natural products and pharmacological agents that are potentially capable of lowering blood glucose levels [22] and attenuating the oxidative stress and inflammation. Under our experimental conditions, Wistar rats treated with a single dose of $40 \mathrm{mg} \mathrm{STZ/kg} \mathrm{bw} \mathrm{underwent} \mathrm{a} \mathrm{marked}$ hyperglycemia (395-416 mg/dL).

The administration of HEYL (100 mg/kg/d) in diabetic animals reduced serum glucose. These results are in agreement with Aybar et al. (2001) [23] and Genta et al. (2010) [24] studies, in which different extracts preparations and doses of Yacon, administered orally, reduced glycemia in STZ-induced diabetic rats, although Raga et al. (2010) [25] demonstrated that a dose of $100 \mathrm{mg} / \mathrm{kg}$ bw of Yacon tea presents more potential activity on glycemic control. The rate of blood glucose reduction in the present study occurred in synergy with the normally functioning pancreatic cells. The DM group without treatment has the lowest concentrations of insulin in plasma. The HEYL promoted a slight increase of insulin concentrations (Figure 1(c)), even without significant difference when compared to untreated DM group, and HOMA-BETA (Figure $1(\mathrm{~d})$ ), suggesting regeneration of functional $\beta$-cells. 


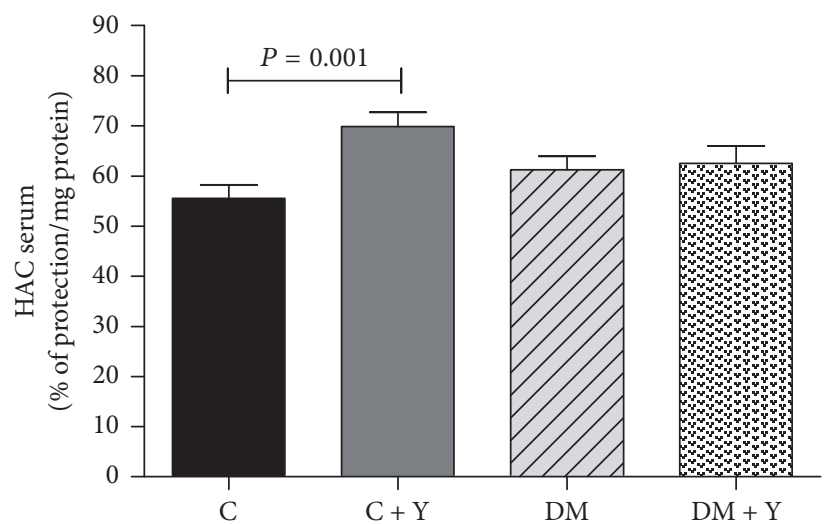

(a)

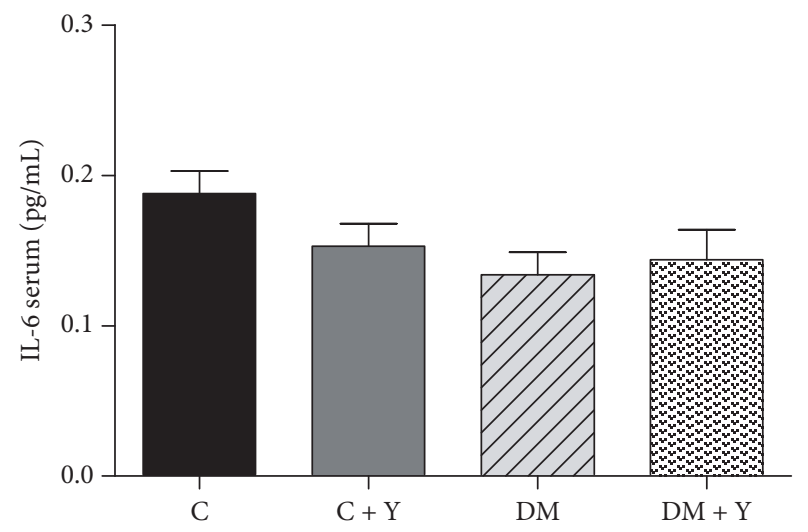

(c)

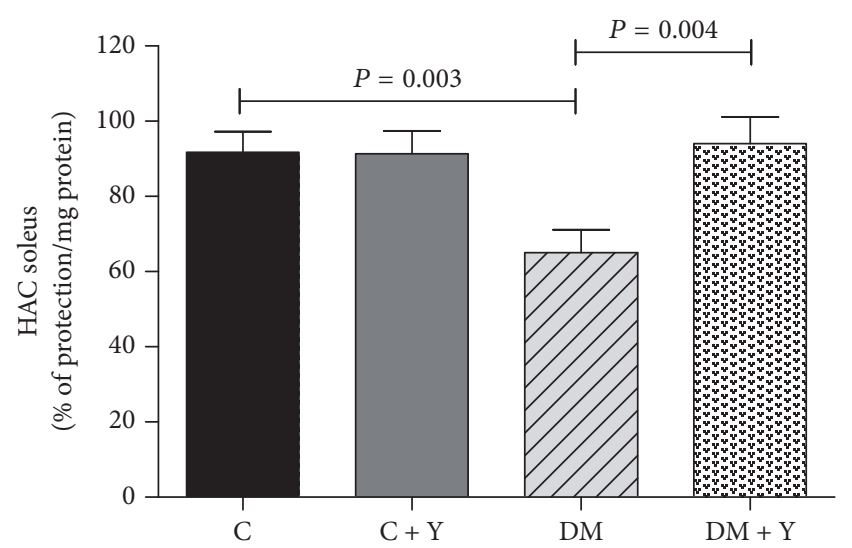

(b)

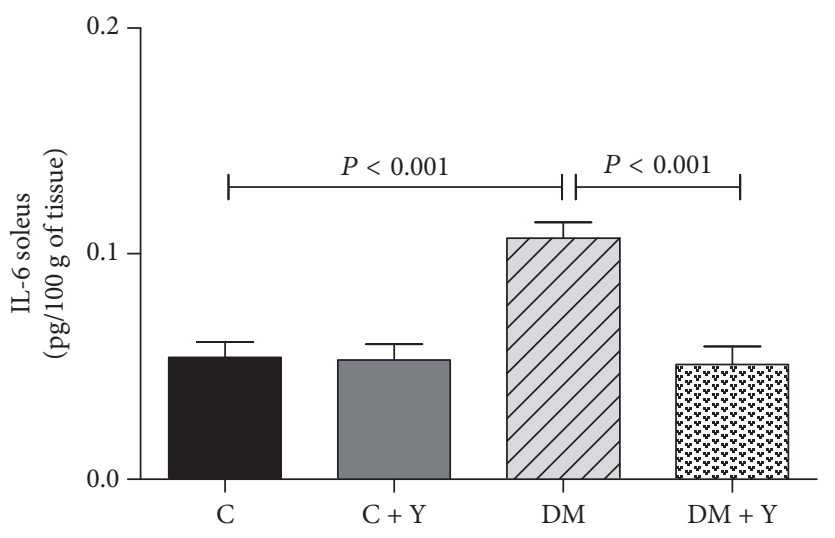

(d)

FIGURE 3: (a) Serum hydrophilic antioxidant capacity (HAC); (b) soleus HAC; (c) serum interleukin-6 (IL-6); (d) soleus IL-6 of the different experimental groups. $\mathrm{C}$ (control group): normal rats; $\mathrm{C}+\mathrm{Y}$ : normal rats receiving HEYL; DM: diabetic rats; and DM + Y: diabetics rats receiving HEYL. The results are expressed as the mean \pm SEM.

It is well known that hyperglycemia is the major cause of diabetic complications. Oxidative stress is one of the potential mechanisms by which hyperglycemia can result in diabetic complications [26]. Improvement of glycemic control that achieves near-normoglycemia can decrease the development and progression of its complications [25]. Regeneration or protection of pancreatic cells that were partially destroyed by STZ with increase of insulin concentrations in plasma and probably increase in the peripheral utilization of glucose could be factors that can explain the significant decrease of fasting blood glucose in the present study [27]. Additionally, some phytochemicals such as flavonoids and polyphenols have been found to be effective due to some other extrapancreatic mechanisms [28]. Further studies are in progress to establish the precise mechanism involved in the antihyperglycemic effect of HEYL.

It has been shown that the solvent used in the preparation of plant extracts can affect positively or negatively the biologically active principles of these plants [8]. Baroni et al. (2008) [29] showed that the hydroethanolic extract of Yacon leaves was the best extraction to promote reduction of glycemia in diabetic and nondiabetic animals. Also, the polyphenolics in Yacon leaves may regulate the free radical activity of STZ diabetes induction [10] and the pathogenesis of diabetes [30]. Plants rich in phenolic compounds have potential hypoglycemic effects $[31,32]$. Ferulic acid, pcoumaric acid, caffeic acid, chlorogenic acid, protocatechuic acid, and quercetin were the highest compounds found in the extract. Jung et al. (2011) [33] found that low doses of onion peel hydroethanolic extract ameliorate hyperglycemia and insulin resistance in high-fat diet/STZ-induced diabetic rats in 8 weeks of treatment. Additionally, Pereira Braga et al. (2013) [34] described that isolated quercetin promotes glucose regulation and decrease of lipid peroxidation in diabetic animals. Caffeic and chlorogenic acids are known for their antioxidant and free radical scavenging properties [35]. Recently, caffeic acid in particular has been associated with reduced blood glucose [36]. Baroni et al. (2016) [37] showed that the phytochemical analysis of the hydroethanolic extract of Yacon identified the presence of phenolic compounds such as caffeic acid, ferulic acid, gallic acid, and chlorogenic acid, corroborating with the present study. The phytochemical profile may explain the antioxidant and antihyperglycemic activities noted in our study.

It is known that the pathogenesis of DM and its complications are associated with the overproduction of ROS and 
depletion of the endogenous antioxidant system, leading to oxidative stress [38]. It is also known that skeletal muscle is a primary tissue in the response to metabolic alteration inducing physiopathological stimulus. Several signaling pathways in striated muscle can be activated by an increase in ROS production [39]. HEYL increased the activity of catalase and GPx (Figure 3) in the soleus muscle is likely attributed to improvement in glucose oxidation or direct modulation of antioxidant enzymes. Some reports suggest that oxidative stress is a key player to diabetic complications, which may be associated with alterations in the metabolism [40, 41]. In addition, it has been reported that STZ induces severe oxidative stress in diabetic animals caused by the peroxidation of polyunsaturated fatty acids, leading to the formation of MDA as by-products of lipid peroxidation [42]. Excessive lipid peroxidation can readily attack the polyunsaturated fatty acids of the lipid membrane, which in turn can disrupt the structure of biological membranes and produce toxic metabolites such as malondialdehyde [43]. MDA is often used as a marker of oxidative damage $[44,45]$. In summary, excess ROS overwhelm antioxidant defenses, leading to oxidative stress.

No significant alterations were found in the plasma of diabetic animals for IL-6 (Figure 2(c)) when compared with controls. Although we did not observe changes in oxidative stress and inflammation markers when they were systemically evaluated after Yacon treatment, the leaves efficiently reduced metabolic markers, such as hyperglycemia, and oxidative/inflammation stress in soleus muscle. In addition, we observed that HAC decreased, while MDA and IL-6 increased in the soleus of diabetic animals (Figure 3), showing the oxidative stress and inflammation in this disorder.

The antioxidant activities of various vegetables, fruits, and plants are mainly attributed to their content of phenolic compounds [46]. The radical scavenging activity of polyphenols depends on the molecular structure and the substitution pattern of the hydroxyl groups, the availability of phenolic hydrogens, and the possibility of stabilization of the resulting phenoxyl radicals via hydrogen donation or by expanded electron delocalization [47]. This radical scavenging ability of extracts could be related to the nature of phenolics, thus contributing to their election transfer/hydrogen donating system.

In the present study, for the first time, the significant increase of the antioxidant status (HAC) and endogenous antioxidant activities (GPx and CAT) and decrease of markers of lipid peroxidation (MDA) and proinflammatory cytokine (IL-6) in soleus muscle in diabetic rats treated with HEYL suggest the antioxidant and anti-inflammatory activity of Yacon extract in this tissue. These results indicate that Yacon leaves have significant effects on scavenging free radicals, promoting decrease of oxidative stress under diabetic conditions.

Although the HEYL promoted several benefits on STZinduced diabetic model, especially those regarding the glucose homeostasis and antioxidant activities, this study has limitations. The precise mechanisms by which HEYL promoted antihyperglycemic activity and increase of insulin concentrations have not been evaluated, even if they are hypothetically attributed to regeneration/preservation of pancreatic beta-cells. But further studies are in progress to investigate the precise mechanism/pathways involved.

STZ administration induces hyperglycemia and increases MDA and IL- 6 in soleus muscle, toxic intermediates in the development of oxidative stress and inflammation in diabetes. Moreover, experimental diabetes decreases the capacity of antioxidant defenses in soleus muscle. In summary, these results demonstrate hyperglycemia-induced oxidative stress in skeletal muscle of diabetic rats. In conclusion, the hydroethanolic extract from S. sonchifolius leaves (HEYL) protects against hyperglycemia, oxidative stress, and inflammation in skeletal muscle and also promotes increase of serum insulin concentrations in STZ-induced diabetic model in rats. These findings provide information that can guide future studies aimed at finding therapeutic alternatives for diabetic complications.

\section{Conflicts of Interest}

The authors declare that there are no conflicts of interest regarding the publication of this paper.

\section{Authors' Contributions}

All authors participated in the design, interpretation of the studies, analysis of the data, and review of the manuscript; Klinsmann Carolo dos Santos and Camila Renata Corrêa elaborated the experimental design; Klinsmann Carolo dos Santos, Bianca Guerra Bueno, Lahis Fernandes Bincoleto, Fabiane Valentini Francisqueti, Lilian Xavier da Silva, Ana Cláudia de Melo Stevanato Nakamune, Lahis Fernandes Bincoleto, and Mariana Gobbo Braz conducted the experiments; Klinsmann Carolo dos Santos, Ana Cláudia de Melo Stevanato Nakamune, C.-Y. Oliver Chen, Jeffrey B. Blumberg, and Camila Renata Corrêa analyzed the data; Klinsmann Carolo dos Santos, Lahis Fernandes Bincoleto, Mariana Gobbo Braz, C.-Y. Oliver Chen, Jeffrey B. Blumberg, and Camila Renata Corrêa revised the manuscript; Klinsmann Carolo dos Santos, C.-Y. Oliver Chen, Jeffrey B. Blumberg, and Camila Renata Corrêa wrote the manuscript.

\section{Acknowledgments}

The authors acknowledge the Coordenação de Aperfeiçoamento de Pessoal de Nível Superior (CAPES), for research grant (PGCI 022/11) and scholar stipend (BEX: 9421/14-9), and Fundação de Amparo à Pesquisa do Estado de São Paulo, Brazil, for financial support (FAPESP, Proc. 2015/10626-0) and scholar stipend (FAPESP, Proc. 2014/17364-8). They also thank Paulo Cesar Georgete and Corina Tomasetti for their technical support and Dr. Átila Francisco Mógor and Dr. Lin Chau Ming for providing the plant material.

\section{References}

[1] P.-T. Yeh, H.-W. Huang, C.-M. Yang, W.-S. Yang, and C.-H. Yang, "Astaxanthin Inhibits Expression of Retinal Oxidative Stress and Inflammatory Mediators in Streptozotocin-Induced Diabetic Rats," PLoS One, vol. 11, no. 1, Article ID e0146438, 2016. 
[2] K. Carolo Dos Santos, C. Pereira Braga, P. Octavio Barbanera, F. Rodrigues Ferreira Seiva, A. Fernandes Jr., and A. A. Henrique Fernandes, "Cardiac energy metabolism and oxidative stress biomarkers in diabetic rat treated with resveratrol," PLOS ONE, vol. 9, no. 7, Article ID e102775, 2014.

[3] T. S. Kern, "Contributions of Inflammatory Processes to the Development of the Early Stages of Diabetic Retinopathy," Experimental Diabetes Research, vol. 2007, Article ID 95103, 2007.

[4] V. Rani, G. Deep, R. K. Singh, K. Palle, and U. C. S. Yadav, "Oxidative stress and metabolic disorders: pathogenesis and therapeutic strategies," Life Sciences, vol. 148, no. 11, pp. 183-193, 2016.

[5] E. Barbieri and P. Sestili, "Reactive Oxygen Species in Skeletal Muscle Signaling," Journal of Signal Transduction, pp. 1-17, 2012.

[6] S. K. Coleman, I. A. Rebalka, DD. M. Souza, and T. J. Hawke, "Skeletal muscle as a therapeutic target for delaying type 1 diabetic complications," World Journal of Diabetes, vol. 6, pp. 1323-1336, 2015.

[7] D. Russo, N. Malafronte, D. Frescura et al., "Antioxidant activities and quali-quantitative analysis of different Smallanthus sonchifolius [(Poepp. and Endl.) H. Robinson] landrace extracts," Natural Product Research, vol. 29, no. 17, pp. 1673-1677, 2014.

[8] B. Simonovska, I. Vovk, S. Andrenšek, K. Valentová, and J. Ulrichová, "Investigation of phenolic acids in yacon (Smallanthus sonchifolius) leaves and tubers," Journal of Chromatography A, vol. 1016, no. 1, pp. 89-98, 2003.

[9] K. Valentová, F. Šeršeň, and J. Ulrichovǎ, "Radical scavenging and anti-lipoperoxidative activities of Smallanthus sonchifolius leaf extracts," Journal of Agricultural and Food Chemistry, vol. 53, no. 14, pp. 5577-5582, 2005.

[10] K. Valentova, L. Cvak, A. Muck, J. Ulrichova, and V. Simanek, "Antioxidant activity of extracts from the leaves of Smallanthus sonchifolius," European Journal of Nutrition, vol. 42, no. 1, pp. 61-66, 2003.

[11] K. Valentová, A. Moncion, I. De Waziers, and J. Ulrichová, “The effect of Smallanthus sonchifolius leaf extracts on rat hepatic metabolism," Cell Biology and Toxicology, vol. 20, no. 2, pp. 109120, 2004.

[12] L. Li, G. Aldini, M. Carini et al., "Characterisation, extraction efficiency, stability and antioxidant activity of phytonutrients in Angelica keiskei," Food Chemistry, vol. 115, no. 1, pp. 227-232, 2009.

[13] D. R. Matthews, J. P. Hosker, A. S. Rudenski, B. A. Naylor, D. F. Treacher, and R. C. Turner, "Homeostasis model assessment: insulin resistance and $\beta$-cell function from fasting plasma glucose and insulin concentrations in man," Diabetologia, vol. 28, no. 7, pp. 412-419, 1985.

[14] D. T. Pierine, M. E. L. Navarro, I. O. Minatel et al., "Lycopene supplementation reduces TNF- $\alpha$ via RAGE in the kidney of obese rats," Nutrition and Diabetes, vol. 4, no. 11, article no. e142, 2014.

[15] G. Beretta, G. Aldini, R. M. Facino, R. M. Russell, N. I. Krinsky, and K.-J. Yeum, "Total antioxidant performance: a validated fluorescence assay for the measurement of plasma oxidizability," Analytical Biochemistry, vol. 354, no. 2, pp. 290-298, 2006.

[16] S. L. Marklund, "Product of extracellular-superoxide dismutase catalysis," FEBS Letters, vol. 184, no. 2, pp. 237-239, 1985.

[17] H. Aebi, "[13] Catalase in vitro," Methods in Enzymology, vol. 105, pp. 121-126, 1984.
[18] L. Flohé and W. A. Günzler, "Assays of glutathione peroxidase," Methods Enzymol, vol. 105, pp. 114-120, 1984.

[19] O. H. Lowry, N. J. Rosebrough, A. L. Farr, and R. J. Randall, "Protein measurement with the Folin phenol reagent," The Journal of Biological Chemistry, vol. 193, pp. 265-275, 1951.

[20] S. Lenzen, "The mechanisms of alloxan- and streptozotocininduced diabetes," Diabetologia, vol. 51, no. 2, pp. 216-226, 2008.

[21] D. Yin, J. Tao, D. D. Lee et al., "Recovery of islet $\beta$-cell function in streptozotocin-induced diabetic mice: an indirect role for the spleen," Diabetes, vol. 55, no. 12, pp. 3256-3263, 2006.

[22] S. Kumar, N. Vasudeva, and S. Sharma, "GC-MS analysis and screening of antidiabetic, antioxidant and hypolipidemic potential of Cinnamomum tamala oil in streptozotocin induced diabetes mellitus in rats," Cardiovascular Diabetology, vol. 11, no. 95, 2012.

[23] M. J. Aybar, A. N. Sánchez Riera, A. Grau, and S. S. Sánchez, "Hypoglycemic effect of the water extract of Smallantus sonchifolius (yacon) leaves in normal and diabetic rats," Journal of Ethnopharmacology, vol. 74, no. 2, pp. 125-132, 2001.

[24] S. B. Genta, W. M. Cabrera, M. I. Mercado, A. Grau, C. A. Catalán, and S. S. Sánchez, "Hypoglycemic activity of leaf organic extracts from Smallanthus sonchifolius: Constituents of the most active fractions," Chemico-Biological Interactions, vol. 185, no. 2, pp. 143-152, 2010.

[25] D. D. Raga, A. B. Alimboyoguen, R. S. Del Fierro, and C. Y. Ragasa, "Hypoglycaemic effects of tea extracts and entkaurenoic acid from Smallanthus sonchifolius," Natural Product Research, vol. 24, no. 18, pp. 1771-1782, 2010.

[26] M. Brownlee, "The pathobiology of diabetic complications: a unifying mechanism," Diabetes, vol. 54, no. 6, pp. 1615-1625, 2005.

[27] W. J. Arion, W. K. Canfield, F. C. Ramos et al., "Chlorogenic acid analogue S 3483: A potent competitive inhibitor of the hepatic and renal glucose-6-phosphatase systems," Archives of Biochemistry and Biophysics, vol. 351, no. 2, pp. 279-285, 1998.

[28] D. K. Patel, S. K. Prasad, R. Kumar, and S. Hemalatha, "An overview on antidiabetic medicinal plants having insulin mimetic property," Asian Pacific Journal of Tropical Biomedicine, vol. 2, pp. 320-330, 2012.

[29] S. Baroni, F. Suzuki-Kemmelmeier, S. M. Caparroz-Assef, R. K. N. Cuman, and C. A. Bersani-Amado, "Effect of crude extracts of leaves of Smallanthus sonchifolius (yacon) on glycemia in diabetic rats," Revista Brasileira de Ciencias Farmaceuticas/Brazilian Journal of Pharmaceutical Sciences, vol. 44, no. 3, pp. 521-530, 2008.

[30] M. M. Gupta and S. Chari, "Lipid peroxidation and antioxidant status in patients with diabetic retinopathy," Indian Journal of Physiology and Pharmacology, vol. 49, pp. 187-192, 2005.

[31] M. Jung, M. Park, H. C. Lee, Y. Kan, E. S. Kang, and S. K. Kim, "Antidiabetic agents from medicinal plants," Current Medicinal Chemistry, vol. 13, no. 10, pp. 1203-1218, 2006.

[32] Y. H. Zhang, J. Y. Cai, H. L. Ruan, H. F. Pi, and J. Z. Wu, "Antihyperglycemic activity of kinsenoside, a high yielding constituent from Anoectochilus roxburghii in streptozotocin diabetic rats," Journal of Ethnopharmacology, vol. 114, no. 2, pp. 141$145,2007$.

[33] J. Y. Jung, Y. Lim, M. S. Moon, J. Y. Kim, and O. Kwon, “Onion peel extracts ameliorate hyperglycemia and insulin resistance in high fat diet/streptozotocin-induced diabetic rats," Nutrition \& Metabolism, vol. 8, no. 18, 2011. 
[34] C. Pereira Braga, A. C. Momentti, F. Barbosa Peixoto et al., "Influence of treatment with quercetin on lipid parameters and oxidative stress of pregnant diabetic rats," Canadian Journal of Physiology and Pharmacology, vol. 91, no. 2, pp. 171-177, 2013.

[35] M. Nardini, F. Natella, V. Gentili, M. D. Felice, and C. Scaccini, "Effect of caffeic acid dietary supplementation on the antioxidant defense system in rat: an in vivo study," Archives of Biochemistry and Biophysics, vol. 342, no. 1, pp. 157-160, 1997.

[36] F.-L. Hsu, Y.-C. Chen, and J.-T. Cheng, "Caffeic acid as active principle from the fruit of Xanthium strumarium to lower plasma glucose in diabetic rats," Planta Medica, vol. 66, no. 3, pp. 228-230, 2000.

[37] S. Baroni, B. A. da Rocha, J. Oliveira de Melo, J. F. Comar, S. M. Caparroz-Assef, and C. A. Bersani-Amado, "Hydroethanolic extract of Smallanthus sonchifolius leaves improves hyperglycemia of streptozotocin induced neonatal diabetic rats," Asian Pacific Journal of Tropical Medicine, vol. 9, no. 5, pp. 432436, 2016.

[38] A. C. Maritim, R. A. Sanders, and J. B. Watkins III, "Diabetes, oxidative stress, and antioxidants: a review," Journal of Biochemical and Molecular Toxicology, vol. 17, no. 1, pp. 24-38, 2003.

[39] K. Sharma, "Mitochondrial hormesis and diabetic complications," Diabetes, vol. 64, no. 3, pp. 663-672, 2015.

[40] G. D. Lopaschuk, J. R. Ussher, C. D. L. Folmes, J. S. Jaswal, and W. C. STANLEY, "Myocardial Fatty Acid Metabolism in Health and Disease. Physiol," Rev, vol. 90, pp. 207-258, 2010.

[41] G. Vassort and B. Turan, "Protective role of antioxidants in diabetes-induced cardiac dysfunction," Cardiovascular Toxicology, vol. 10, no. 2, pp. 73-86, 2010.

[42] T. Mahesh and V. P. Menon, "Quercetin Allievates Oxidative Stress in Streptozotocin-induced Diabetic Rats," Phyther. Res, vol. 18, pp. 123-127, 2004.

[43] H. J. He, G. Y. Wang, Y. Gao, W. H. Ling, Z. W. Yu, and T. R. Jin, "Curcumin attenuates Nrf2 signaling defect, oxidative stress in muscle and glucose intolerance in high fat diet-fed mice," World Journal of Diabetes, vol. 3, no. 5, pp. 94-104, 2012.

[44] R. Mittal, S. Sharma, S. Chhibber, and K. Harjai, "Evaluation of interleukin-10 production in Pseudomonas aeruginosa induced acute pyelonephritis," Journal of Infection and Public Health, vol. 2, no. 3, pp. 136-140, 2009.

[45] A. Arya, C. Yeng Looi, S. Chuen Cheah, M. Rais Mustafa, and M. Ali Mohd, "Anti-diabetic effects of Centratherum anthelminticum seeds methanolic fraction on pancreatic cells, $\beta$-TC6 and its alleviating role in type 2 diabetic rats," Journal of Ethnopharmacology, vol. 144, no. 1, pp. 22-32, 2012.

[46] C.-Y. O. Chen, A. Kamil, and J. B. Blumberg, "Phytochemical composition and antioxidant capacity of whole wheat products," Int. J. Food Sci. Nutr, vol. 66, pp. 63-70, 2015.

[47] V. Benković, N. Kopjar, A. Horvat Kneževic et al., "Evaluation of radioprotective effects of propolis and quercetin on human white blood cells in vitro," Biological and Pharmaceutical Bulletin, vol. 31, no. 9, pp. 1778-1785, 2008. 


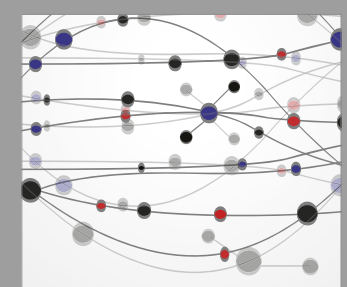

The Scientific World Journal
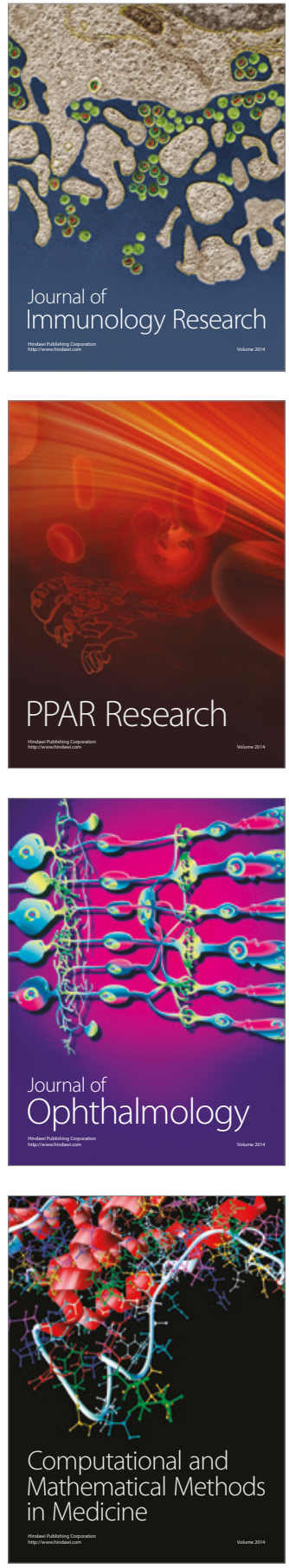

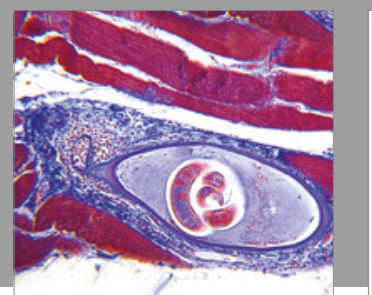

Gastroenterology Research and Practice
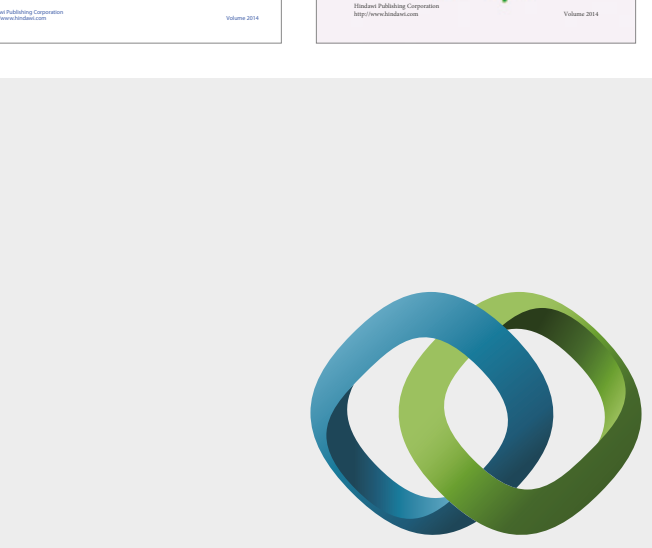

\section{Hindawi}

Submit your manuscripts at

https://www.hindawi.com
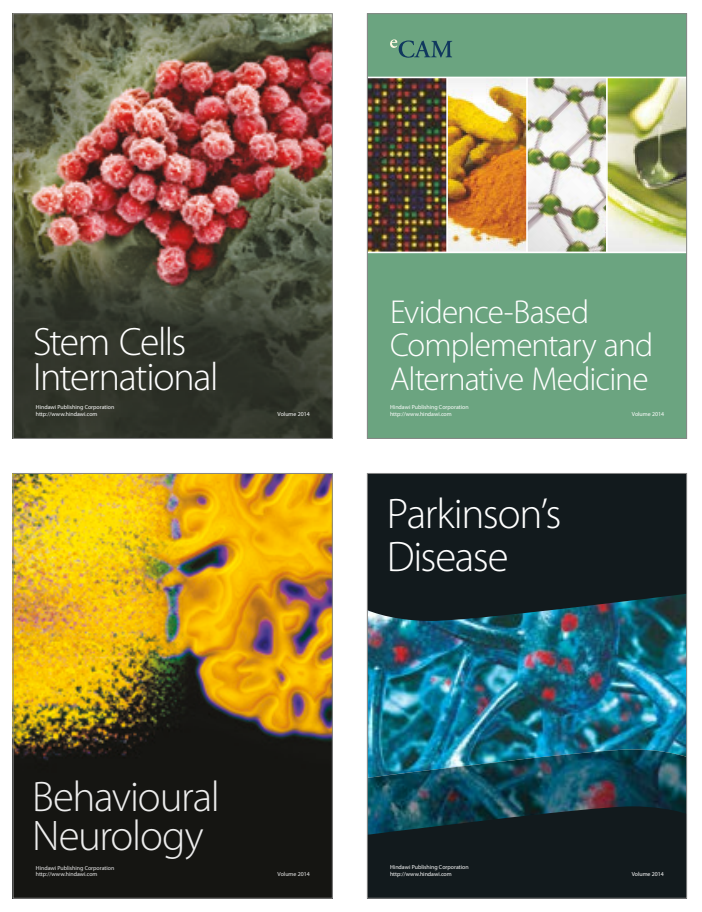
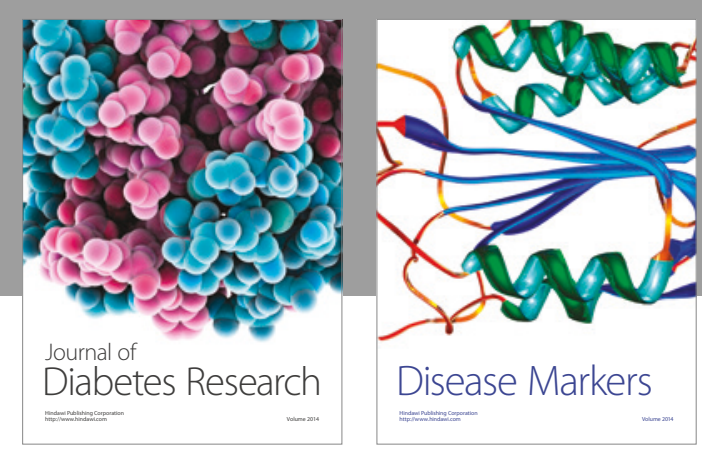

Disease Markers
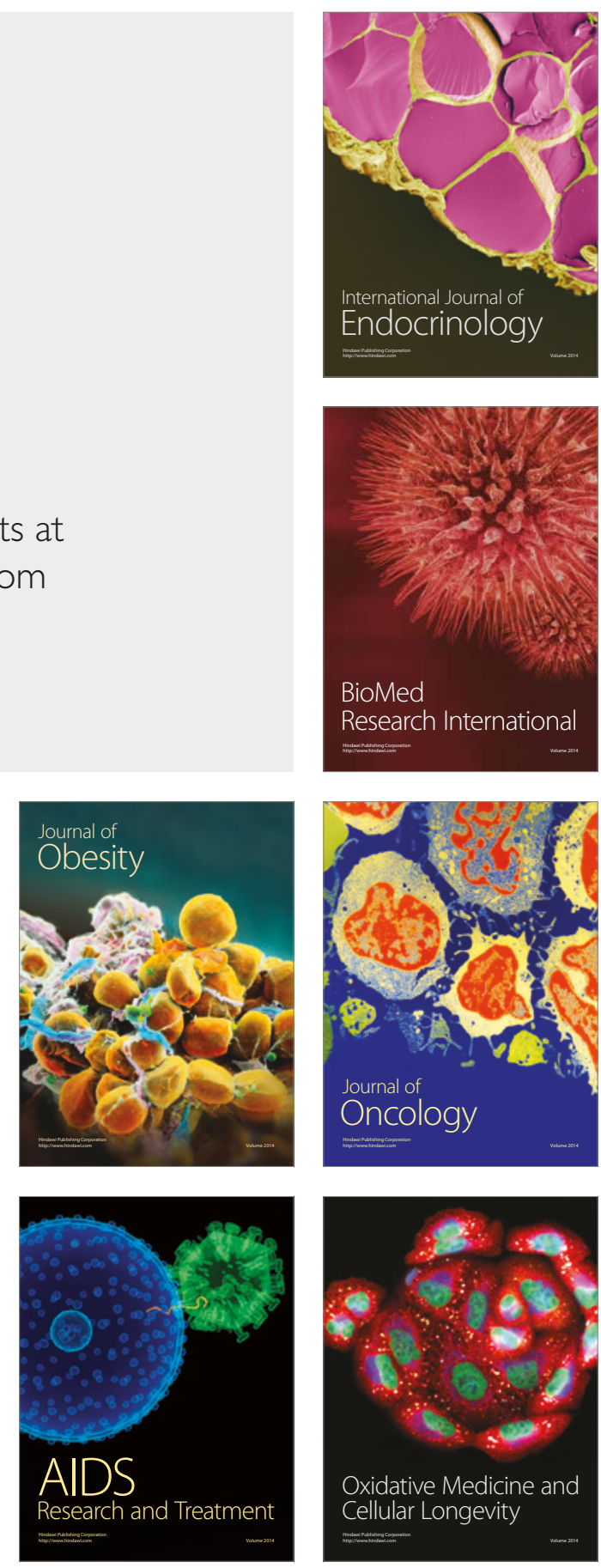